\title{
Development of 3-5 THz Harmonic Mixer
}

\author{
B.T. Bulcha ${ }^{1}$, J.L. Hesler ${ }^{2}$, V. Drakinskiy ${ }^{4}$, J. Stake ${ }^{4}$, N.S. Barker ${ }^{3}$ \\ ${ }^{1}$ NASA Goddard Space Flight Center, Greenbelt, MD, 20771, USA., ${ }^{2}$ Virginia Diodes, Inc., Charlottesville, VA, 22902, USA. \\ ${ }^{3}$ University of Virginia, Charlottesville, VA, 22902, USA., ${ }^{4}$ Chalmers University of Technology, Göteborg, Sweden.
}

\begin{abstract}
There is a high need for $>2$ THz room temperature direct-detectors/harmonic-mixers to characterize THz optical sources, phase lock THz-QCLs as LO sources for multi-pixel receivers, and realize absolute frequency calibration sources for applications in astrophysics, earth science, and remote sensing. Thus, we have developed a WM-52 (WR-0.22) harmonic mixer for the 3 - 5 THz operation. The design, fabrication and assembly of the THz mixer are discussed.
\end{abstract}

\section{INTRODUCTION}

THz technology spanning the Far-Infrared (FIR) and SubMillimeter (SMM) wavelengths has applications in astrophysics, earth and planetary science and a stabilized Local Oscillator (LO) is a critical component to build Terahertz (THz) instruments that enable airborne and ground based science investigations [4][6]. Compact and stable $\mathrm{THz}$ receivers can be mounted on a spacecraft, aircraft, balloon or in a cubesat to measure dynamics in the atmosphere, trace gas distribution, radiation balance and thermal structure [5]. Due to intervening dusty materials in space, it is difficult to use visible-light telescopes to perform astrophysical measurements to address key questions such as the process of star formation in our galaxy and in galaxies of the earlier universe. However, the effect of obscuration in FIR and SMM wavelength regions is nearly negligible and probing star forming region dynamics and content via high-resolution spectroscopy is feasible using $\mathrm{THz}$ instruments.

Therefore, a high-output-power and highly stable $2-6 \mathrm{THz}$ Local Oscillator (LO) with low phase noise is necessary to build a multi-pixel receiver $(\mathrm{n}>10)$, a tunable heterodyne spectrometer and an interferometer with high spectral resolution $\left(R=\lambda / \Delta \lambda>10^{7}\right)$ and $<\mathrm{kHz}$ long term stability. This can be achieved by phase locking a Quantum Cascade Laser (QCL) to a highly stable microwave reference to produce a high power and stable LO source using a room temperature harmonic mixer [3].

\section{THZ HARMONIC MIXER}

Previously, we demonstrated a $2-3.2 \mathrm{THz}$ harmonic mixer with a conversion loss of $\sim 27 \mathrm{~dB}$ and used the mixer to phase lock a $2.5 \mathrm{THz}$ and $2.7 \mathrm{THz}$ QCLs [2]. To realize such an implementation at higher frequencies, we designed and fabricated a WR-0.22 harmonic mixer with GaAs-based substrate, and thickness of $2 \mu \mathrm{m}$ and width of $18 \mu \mathrm{m}$. The integrated $\mathrm{THz}$ circuit is fabricated at Chalmers University and the diode process is based on electron beam lithography, with a beam spot of less than $5 \mu \mathrm{m}$, allowing precise and repeatable anode and air bridge formation [1]. Anode areas of $\sim 0.28 \mu \mathrm{m}^{2}$ were fabricated for approximately $3-4 \mu \mathrm{m}$ finger length structures. The mixer also contained a WR-1.0 LO waveguide that allows $750-1100 \mathrm{GHz}$ signal and couples to the quartz based LO probe. To maintain the LO termination, the transmission line length is kept a half wavelength away from the diode to the short circuited beam lead. When the mixer is pumped through the LO port, it is important to deliver maximum power by isolating the $\mathrm{LO}$ and IF channel; therefore, an IF circuit containing a Hi-Z and Low-Z based low pass filter is used to block the LO signal propagation into the IF channel. Fig. 1 depicts the internal circuitry and the machined block of the WR-0.22 harmonic mixer.

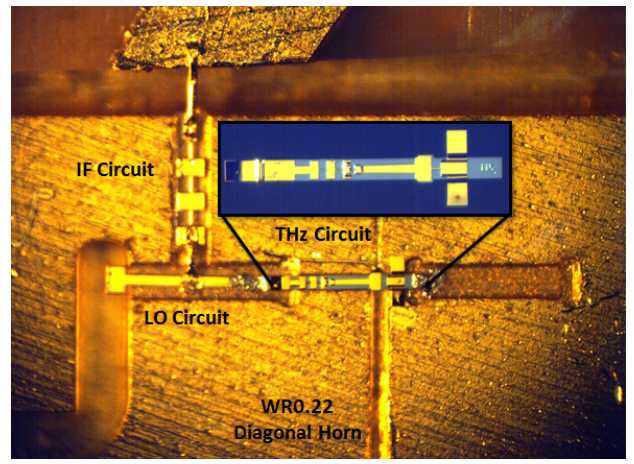

Figure 1. WM-52 (WR-0.22) harmonic mixer design.

Machining the mixer's block is performed using $\mathrm{CNC}$ milling machine. The block has an E-plane split between the base and the cover, and the base contained the $\mathrm{THz}$ channel, which is the smallest dimensions, approximately $6 \mu \mathrm{m}$ by $26 \mu \mathrm{m}$. Due to the smaller dimensions, assembling the circuits by hand within $2-3 \mu \mathrm{m}$ accuracy is a very challenging task to achieve. For the targeted design of a $\mathrm{THz}$ circuit floating height of $6 \mu \mathrm{m}$, machining error in the $\mathrm{THz}$ circuit channel could introduce $2-3 \mu \mathrm{m}$ variation which can leads to a $3-9 \mu \mathrm{m}$ final floating height. The mixer is assembled at Virginia Diodes, Inc. under a microscope. First the mixer block is cleaned properly, followed by mounting the LO probe, IF filter, IF circuit and THz supporting substrate circuits into their proper channels. Bonding the LO probe with the IF circuit and IF filter with IF circuit is done using $16 \mu \mathrm{m}$ and $24.5 \mu \mathrm{m}$ bond wires respectively. Once those circuits are stably positioned, the $\mathrm{THz}$ circuit is placed on top for the LO probe and the THz supporting circuit and connected using conductive epoxy. Once all the circuits are mounted in a mixer block as shown in Fig. 1, the two halves of the block are aligned, fastened together and the block is ready for RF testing. 


\section{INITIAL CHARACTERIZATION}

The initial characterization of the mixer is performed by testing the mixer's current-voltage (I-V) relationship (Fig.2), and the diode DC-parameters are extracted by curve fitting to an ideal diode model. The diode parameters are series resistance $\mathrm{R}_{\mathrm{S}}=25 \Omega$, ideality factor $\eta=1.45$ and saturation current $\mathrm{I}_{0}=13 \mathrm{fA}$.

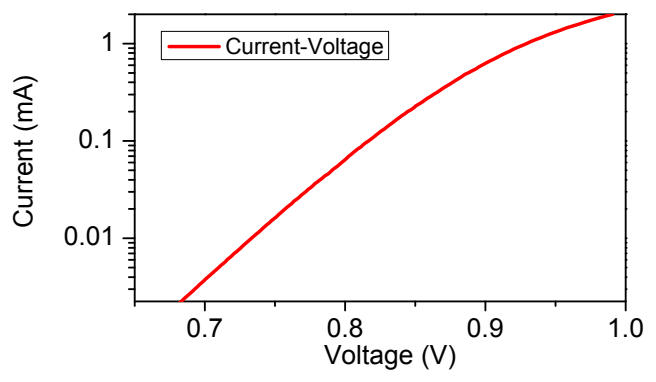

Figure 2. I-V curve of the $\mathrm{THz}$ diode.

To realize the LO coupling efficiency, the mixer's return loss is measured using VDI's WR-1.0 extender modules for 750$1100 \mathrm{GHz}$. The systematic errors are removed by calibrating the extender modules using VDI's standard TRL calibration kit. The return loss measurement is repeated for different current biases between 16-275 $\mu \mathrm{A}$. The performance improved for higher current biases, and the test result shows a nominal return loss of $10 \mathrm{~dB}$ for frequencies above $900 \mathrm{GHz}$, Fig. 3 .

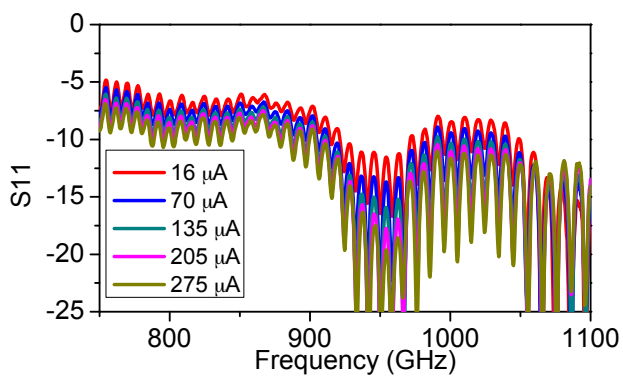

Figure 3. LO return loss for $750-1100 \mathrm{GHz}$ using WR-1.0 extender module.

LO video response at $830 \mathrm{GHz}$ is measured actively for a range of input powers by pumping the mixer with $\mathrm{LO}$ power while modulating the LO signal and measuring the output voltage on a lock-in amplifier. To perform this measurement, a high power WR-1.0 amplifier multiplier chain (AMC) is used to drive the mixer and a coupler is used to actively measure the input power to the mixer. The mixer is also current biased at $16 \mu \mathrm{A}$ and $135 \mu \mathrm{A}$ and the LO-responsivity of the mixer is estimated to be $500 \mathrm{~V} / \mathrm{W}$. Due to the relatively improved return loss performance above $900 \mathrm{GHz}$, the $\mathrm{LO}$ video response and responsivity are expected to perform more than $500 \mathrm{~V} / \mathrm{W}$.

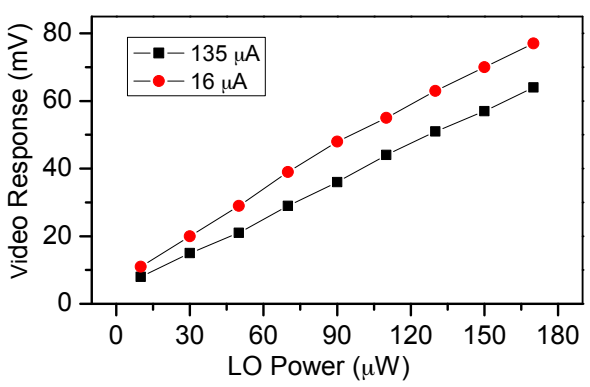

Figure 4. LO video response vs. input power for current bias of $16 \mu \mathrm{A}$ and $135 \mu \mathrm{A}$.

From the HFSS model, the RF probe has a return loss of greater than $15 \mathrm{~dB}$. Moreover, the mixer's RF responsivity and conversion loss performance will be tested using $3.8 \mathrm{THz}$ and 4.7 THz QCLs. The mixer will be utilized to phase lock the QCLs to use as stable frequency source and $\mathrm{THz} \mathrm{LO}$ oscillator.

\section{CONCLUSION AND FUTURE WORKS}

A WR-0.22 harmonic mixer is designed and fabricated to phase lock QCLs. Once the mixer is assembled, the initial performance, such as I-V, LO return loss and LO video response at different current biases are measured. Future work includes characterizing the RF video response, conversion loss of the mixer and phase locking of QCLs within the WR- 0.22 harmonic mixer band, particularly $3.8 \mathrm{THz}$ and $4.7 \mathrm{THz}$.

\section{ACKNOWLEDGMENT}

The 3-5 THz mixer design, fabrication, assembly and testing were supported by Virginia Diode. Inc, University of Chalmers and University of Virginia.

\section{REFERENCES}

[1] A.Y Tang, V. Drakinskiy, P. Sobis, J. Vukusic, J. Stake. "Modeling of GaAs Schottky diodes for terahertz application". In 34th International Conference on Infrared, Millimeter, and Terahertz Waves (IRMMW-THz), pages 1-2, September 2009.

[2] Berhanu T. Bulcha; Jeffrey L. Hesler; Vladimir Drakinskiy; Jan Stake; Alex Valavanis; Paul Dean; Lianhe H. Li; N. Scott Barker. "Design and Characterization of 1.8-3.2 THz Schottky Based Harmonic Mixers". IEEE Transactions on Terahertz Science and Technology, Volume: 6, no. 5:pages: 737 - 746, September 2016.

[3] B.T. Bulcha, J.L. Hesler, A. Valavanis, V. Drakinskiy, J. Stake, R. Dong, J.X. Zhu, P. Dean, L.H. Li, A.G. Davies, E.H. Linfield, N.S. Barke. "Phase locking of a $2.5 \mathrm{THz}$ Quantum Cascade Laser to a microwave reference using THz Schottky mixer". 40th International Conference on Infrared, Millimeter, and Terahertz waves (IRMMW-THz), pages 1-2, September 2015.

[4] G.Chain. "Optical pumped submillimeter laser heterodyne receivers astrophysical observation and recent technological development". Proceedings of the IEEE, Volume: 80, no. 11:pages: 1788 - 1799, 1992.

[5] L. Schrottke, M. Wienold, R. Sharma, X. Lu, K. Biermann, R. Hey, A. Tahraoui, H. Richter, H. W. Hubers, H.T. Grahn. "Quantum-cascade lasers as local oscillators for heterodyne spectrometers in the spectral range around 4.745 THz". Semiconductor Science and Technology, Volume 28, no. 3, February 2013.

[6] Martin Harwit, George Helou, Lee Armus, C. Matt Bradford, Paul F. Goldsmith, Michael Hauser, David Leisawitz, Daniel F. Lester, George Rieke, and Stephen A. Rinehart. "Far-Infrared/Submillimeter Astronomy from Space Tracking an Evolving Universe and the Emergence of Life". Technical report, The Astronomy \& Astrophysics Decadal Survey, 2010. 